\title{
Full Duplex Spatial Modulation System in presence of IQ imbalance
}

\author{
Yanni Zhou, Florin Hutu and Guillaume Villemaud \\ Univ Lyon, INSA Lyon, Inria, CITI, F-69621, Villeurbanne, France
}

\begin{abstract}
The combination of the spatial modulation (SM) and of the full duplex (FD) is a challenging research direction because it brings high spectral efficiency and even energy consumption reduction because of the use of a unique radio frequency (RF) receiving chain. This paper presents a fullduplex spatial modulation (FDSM) system over the Rician fading channel. A least-square self-interference (SI) estimator is implemented for the self-interference cancellation (SIC). Moreover, the impact of IQ imbalance on the bit error rate (BER) performance is analyzed. The obtained results show that the proposed estimator can reduce SI at a low level. The system is more sensitive to the IQ imbalance under low noise environment.
\end{abstract}

\section{Introduction}

Multiple-Input Multiple-Output (MIMO) transmission technology has attracted our attention because of its capacity and diversity gains. However, the complexity and cost of MIMO systems are the critical factors that limit their development. Spatial modulation (SM) as a new MIMO technology has been proposed to solve these problems. Only one RF chain is needed for SM, which avoids high interchannel interference and spectrum congestion. As the information bits are transmitted by both the index of the activated antenna and traditional constellation modulation, the spectral efficiency is guaranteed. A complete SM system with a channel state information (CSI) detector over the Rician fading channel has been studied in [1].

The current studies of SM systems [2] [3] mainly focus on the half-duplex mode. The combination of the SM and FD is a future research direction which can enable to the communication nodes to transmit and receive simultaneously and at the same frequency. However, the FD system suffers from strong self-interference. Because a single RF chain is implemented, the self-interference cancellation (SIC) complexity will not increase, but the spectral efficiency is doubled. The superiority of the full duplex spatial modulation (FDSM) system is discussed in [4] and [5].

In practice, the silicon implementation of the RF receiver will undoubtedly cause in-phase and quadrature (IQ) imbalance. IQ imbalance is the mismatch of the amplitude and the phase between I and Q branches, which can lead to a degradation in system performance. Besides, IQ imbalance has been considered in FD system in [6] and [7]. To the best of our knowledge, no work has been done to understand the IQ imbalance impact on the FDSM systems.

In this paper, a $2 \times 2$ FDSM system over the Rician fading channel is studied. The elaborate receiver structure in the presence of IQ imbalance is designed in this FDSM system. The framework of the proposed system is implemented in Keysigth's Advanced Design System (ADS). Moreover, Matlab co-simulation allows the implementation of SIC algorithm.

The reminder of this paper is organized into three sections: in section II, we introduce the structure of a $2 \times 2$ FDSM system model. Section III gives the simulation results in two parts: one concerning the performance of the proposed SI detector with ideal IQ, the other highlights the impact of the IQ imbalance on the bit error rate (BER) performance. Section IV concludes this paper and gives the future directions.

\section{System model}

Consider that both nodes of the FDSM system are equipped with the transmit and receive capacities. The structure of the node A is shown in Figure 1. The basic idea of the SM system is that the information bits are transmitted not by the tradition amplitude and phase modulation but also by the index of the activated transmit antenna.

At each transmission interval, $\log _{2}\left(N_{t} M\right)$ information bits are emitted. Therefore, we divide the incoming bitstream into groups of $\log _{2}\left(N_{t} M\right)$ bits. $N_{t}$ represents the number of transmit antennas, $M$ represents the corresponding modulation order. The transmit antenna is selected according to the first $\log _{2}\left(N_{t}\right)$ bits, the remaining $\log _{2}(M)$ bits are forming the symbol of the quadrature amplitude modulation (QAM) modulation.

In our case, quadrature phase-shift keying (QPSK or 4QAM) modulation is implemented in a $2 \times 2$ FDSM system, so $M=4, N_{t}=2$. Then, the modulated symbol is transmitted through a $2 \times 2$ sized Rician fading channel model $\mathbf{H}$, which is also influenced by an AWN noise $\eta$. The 


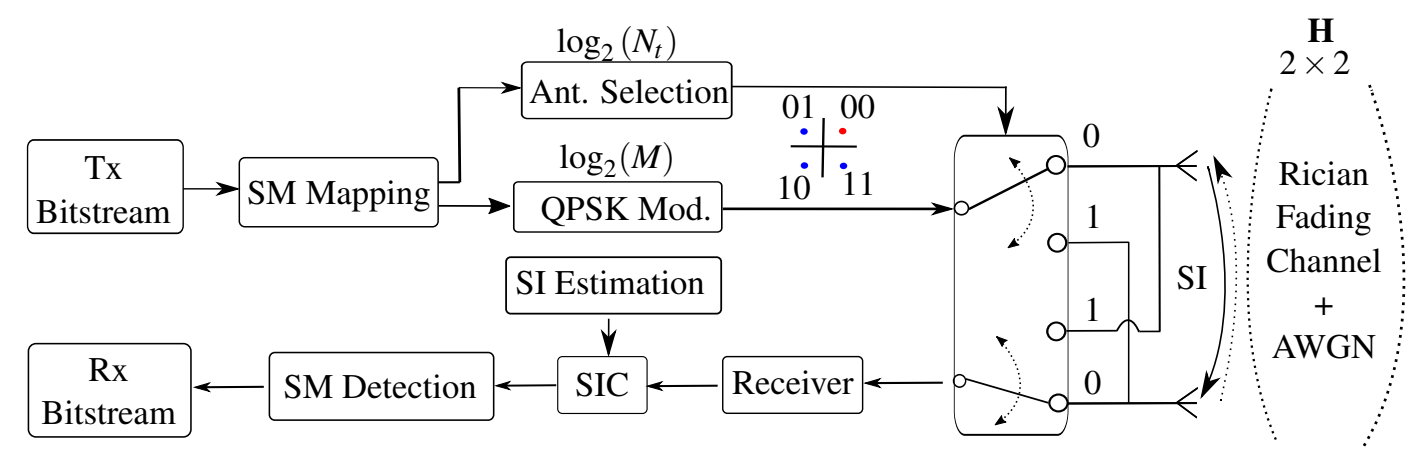

Figure 1. $2 \times 2$ Full Duplex Spatial Modulation system: node A

channel matrix $\mathbf{H}$ is defined as equation (1).

$$
\begin{gathered}
\mathbf{H}=\sqrt{\frac{K}{K+1}} \cdot \mathbf{H}_{\mathbf{L O S}}+\sqrt{\frac{1}{K+1}} \cdot \mathbf{H}_{\mathbf{N L O S}} \\
\mathbf{y}=\mathbf{H} \cdot \mathbf{x}+\eta
\end{gathered}
$$

where $\mathbf{H}_{\mathbf{L O S}}$ and $\mathbf{H}_{\mathbf{N L O S}}$ represent the line-of-sight (LOS) and non-line-of-sight (NLOS) components of $\mathbf{H}$, respectively. $K$ represents the Ricean factor. A larger value of $K$ indicates a stronger channel correlation.

The receiving antenna at node $\mathrm{A}$ will receive not only the useful signal from Node B but also the strong selfinterference which is emitted by the neighbor selected antenna. Consider the signals issued from the I and $\mathrm{Q}$ branches of the receiver $S_{i}$ and $S_{q}$ respectively, where $S_{i n}$ is the received signal.

$$
\begin{aligned}
& S_{i}^{*}=\left(1+\frac{\varepsilon}{2}\right) \cdot \sin \left(2 \pi f_{c} t+\frac{\theta}{2}\right) \cdot S_{i n} \\
& S_{q}^{*}=\left(1-\frac{\varepsilon}{2}\right) \cdot \cos \left(2 \pi f_{c} t-\frac{\theta}{2}\right) \cdot S_{i n}
\end{aligned}
$$

The quantities $\varepsilon$ and $\theta$ are the amplitude imbalance and phase imbalance between the I and Q chains, respectively. $f_{c}$ is the carrier frequency of the received signal.

Moreover, a least-square estimator is proposed as SIC algorithm. We assume that CSI can be wholly achieved at the receiving end. The principle of this estimator is adding a sequence of $N$ test bits as the head of the incoming bit. SI estimation channel $H_{S I}$ is calculated by detecting the relationship between the received signal $\mathbf{y}$ and the input signal $\mathbf{x}$.

$$
H_{S I}=\frac{1}{N^{2}} \cdot\left[\mathbf{x}^{\mathbf{t}} \cdot \mathbf{x}^{\mathbf{t}^{H}}\right]^{-1} \cdot \overline{\mathbf{y}^{\mathbf{t}}} \cdot \mathbf{x}^{-1}
$$

The operator $[\cdot]^{t}$ represents the transpose, $[\cdot]^{H}$ denotes the Hermitian transpose and $\overline{[\cdot]}$ indicates the complex conjugate.

Finally, the index of the transmitting antenna is detected based on the channel state information (CSI) to recover the input bitstream. A Rician fading channel correction depending on CSI is also envisaged for the QPSK demodulation. CSI detection method is presented in our previous work [1].

\section{Simulation results}

\subsection{SIC without IQ imbalance}

The BER performance of the FDSM system with different SIC levels is shown in Figure 2. When no SIC is achieved, one can remark that the BER remains at around 0.5 for selfinterference-to-noise ratio $(\mathrm{INR})=20 \mathrm{~dB}$ even though the signal-to-noise ratio per bit $\left(E_{b} / N_{0}\right)$ reach $20 \mathrm{~dB}$. The number of test bits, $N$ is the main factor that affects the accuracy of SI estimation. It can be concluded that the increase of $\mathrm{N}$ (from 10 to 1000 ) can eliminate SI with an original INR = $20 \mathrm{~dB}$ to a level even below INR $=-10 \mathrm{~dB}$.

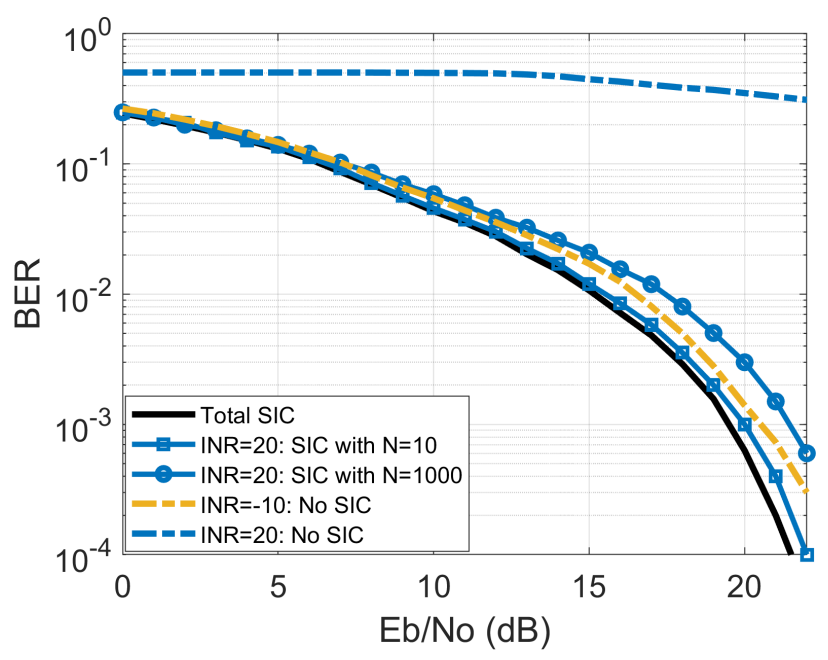

Figure 2. Comparison of the BER performance of the FDSM system with different SIC levels (total SIC, no SIC, SIC with 10 ou 1000 test numbers) 


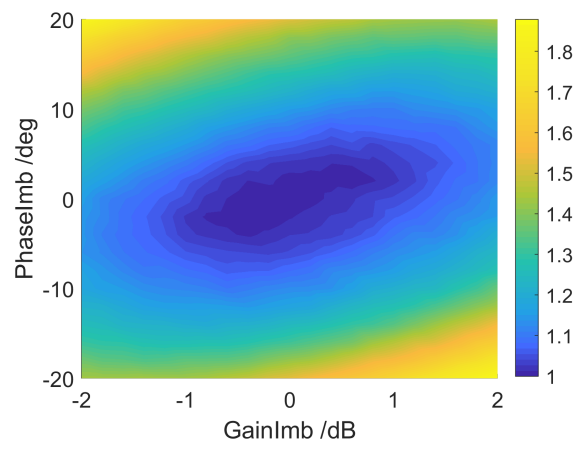

(a) $\mathrm{Eb} / \mathrm{No}=10 \mathrm{~dB}$

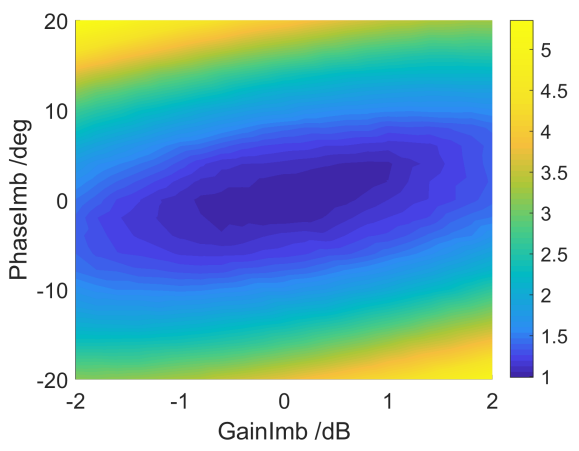

(b) $\mathrm{Eb} / \mathrm{No}=15 \mathrm{~dB}$

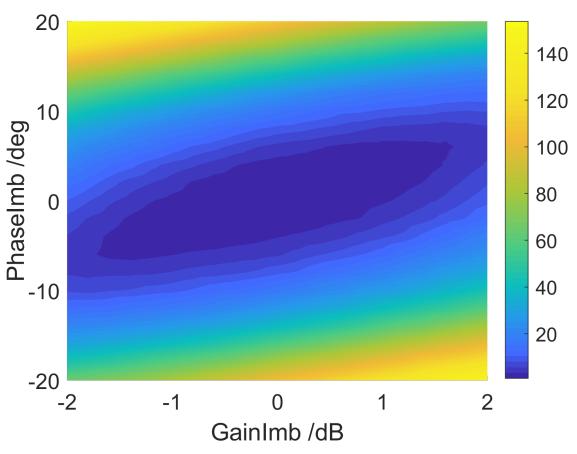

(c) $\mathrm{Eb} / \mathrm{No}=20 \mathrm{~dB}$

Figure 3. Relative BER of the FDSM system in presence of IQ imbalance under $\mathrm{Eb} / \mathrm{No}=10,15$ and $20 \mathrm{~dB}$

\subsection{Impact of IQ imbalance under different noise levels}

The relative BER is the ratio of the BER actual with IQ imbalance and the BER with ideal IQ. The gain imbalance IQ varied from $-2 \mathrm{~dB}$ to $2 \mathrm{~dB}$; and the phase imbalance is varied from $-20^{\circ}$ to $20^{\circ}$. The gain imbalance can be defined as $G_{\text {imb }}= \pm 10 \cdot \log _{10}(\varepsilon)$. Three different spectral noise density environments have been studied for BER performance. It can be observed from Figure 3 that the system is more sensitive to IQ imbalance as $E_{b} / N_{0}$ increases. In the same range, when $E_{b} / N_{0}=20 \mathrm{~dB}$, relative BER can reach up to 150 compared with less than 2 under $E_{b} / N_{0}=20 \mathrm{~dB}$. The reason is that the thermal noise when $E_{b} / N_{0}$ is small can degrade the performance, so the addition of IQ imbalance will not make a great difference. The case is opposite with high $E_{b} / N_{0}$.

\section{Conclusion}

In this paper, we have presented a $2 \times 2$ FDSM system over the Rician fading channel. A SI estimator for SIC and a receiver with IQ imbalance have been presented. Based on the ADS-Matlab co-simulation results, it can be concluded that the proposed estimator can maintain SIC to an acceptable level without IQ imbalance. The IQ imbalance has more impact on the BER performance under high spectral noise density levels. Further study will focus on the estimation and compensation of IQ imbalance. The other RF impairments such as phase noise will also be considered.

\section{References}

[1] Y. Zhou, F. Hutu, and G. Villemaud, "Analysis of a Spatial Modulation System over Time-varying Rician Fad- ing Channel with a CSI Detector," in IEEE Radio and Wireless Symposium (RWS), 2020.

[2] M. D. Renzo and H. Haas, "Performance Analysis of Spatial Modulation," in IEEE International Conference on Communications and Networking, 2010.

[3] M. D. Renzo, H. Haas, A. Ghrayeb, S. Sugiura, and L. Hanzo, "Spatial Modulation for Generalized MIMO: Challenges, Opportunities, and Implementation," Proceedings of the IEEE, vol. 102, no. 1, pp. 56 - 103, 2013.

[4] J. Zhang, Q. Li, K. J. Kim, Y. Wang, X. Ge, and J. Zhang, "On the Performance of Full-Duplex TwoWay Relay Channels with Spatial Modulation," IEEE Transactions on Communications, vol. 64, no. 12, pp. 4966-4982, 2016.

[5] S. Narayanan, H. Ahmadi, and M. F. Flanagan, "Simultaneous Uplink/Downlink Transmission Using FullDuplex Single-RF MIMO," IEEE Wireless Communications Letters, vol. 5, no. 1, pp. 88-91, feb 2016.

[6] H. Yu, F. Shu, Y. You, J. Wang, T. Liu, X. You, J. Lu, J. Wang, and X. Zhu, "Compressed sensingbased time-domain channel estimator for full-duplex OFDM systems with IQ-imbalances," Sci. China Inform. Sci, vol. 60, no. 8, p. 082303, 2017.

[7] Z. Zhan, G. Villemaud, F. Hutu, and J.-M. Gorce, "Digital Estimation and Compensation of I / Q Imbalance for Full-Duplex Dual-Band OFDM Radio," in IEEE 25th Annual International Symposium on Personal, Indoor, and Mobile Radio Communication (PIMRC), 2014. 